\title{
Suboptimal Control Using Model Order Reduction
}

\author{
Avadh Pati, Awadhesh Kumar, and Dinesh Chandra \\ Electrical Engineering Department, Motilal Nehru National Institute of Technology, Allahabad, India
}

Correspondence should be addressed to Avadh Pati; er.avadhmnnit@gmail.com

Received 27 September 2013; Accepted 19 December 2013; Published 3 February 2014

Academic Editors: X. Ma and J. Yu

Copyright (C) 2014 Avadh Pati et al. This is an open access article distributed under the Creative Commons Attribution License, which permits unrestricted use, distribution, and reproduction in any medium, provided the original work is properly cited.

\begin{abstract}
A Padé approximation based technique for designing a suboptimal controller is presented. The technique uses matching of both time moments and Markov parameters for model order reduction. In this method, the suboptimal controller is first derived for reduced order model and then implemented for higher order plant by partial feedback of measurable states.
\end{abstract}

\section{Introduction}

The design of an optimal control law for large scale systems is inconvenient due to one of the main drawbacks of optimal control theory that requires feedback from all state variables that are defined to describe the dynamics of the plant. It is rare that all the states are available for measurement [1] and if one tries to design optimal controller, Kalman filter or state observer is required to reconstruct the missing state of the plant. This introduces the high order dynamics in the control function and leads to a complicated and costly controller. In order to minimize this problem, an attempt has been made for the design of incomplete state feedback suboptimal control law using measurable states [2-4]. In this paper a method for suboptimal controller design is proposed. The suboptimal control can be achieved by using model order reduction technique for higher order system.

The plant (higher order system) can be reduced by Padé approximation technique (matching of both time moments and Markov parameters) into reduced order model, and then, with the help of reduced order controller, one can design the suboptimal controller for plant (higher order systems). The usefulness of techniques for deriving reduced order approximations of high order system has been already accepted due to the advantages of reduced computational effort and increased understanding of original system $[5,6]$. Consequently, a large number of time-domain (Hankel norm, balance truncation, and Krylov subspace projection) and frequency-domain (Moment matching, Padé approximation, Routh-Padé approximation, and pole clustering methods) system simplification techniques have been developed to suit the different requirements and a variety of numerical approximation are applied to obtain the reduced order model [7]. In this paper the plant (higher order system) is reduced by Padé approximation technique and suboptimal controller is derived with the reduced order model. Then the suboptimal controller is implemented for original plant (higher order system). This reduces the computational complexity of controller design. Two numerical examples are included to illustrate the procedure.

\section{The Design Procedure}

Consider a single-input single-output linear ( $n$ th-order) dynamic system described by

$$
\begin{gathered}
\dot{x}=A x(t)+B u(t), \\
y=C x(t),
\end{gathered}
$$

with the quadratic cost function

$$
j=\int_{0}^{\infty}\left\{x^{T}(t) Q x(t)+R u^{2}(t)\right\},
$$

where $Q$ is the $(n \times n)$ positive semidefinite matrix and $R$ is a positive weight. $A, B$, and $C$ are matrices of appropriate dimensions. It is well know that the optimal feedback control law is linear combination of the state variables. A block diagram of the plant using control law has been shown in Figure 1. 


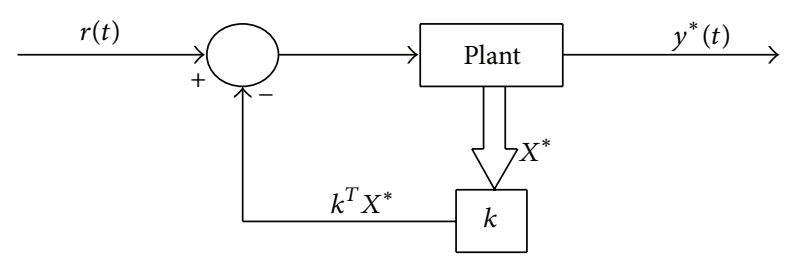

FIGURE 1: Block diagram of controlled system.

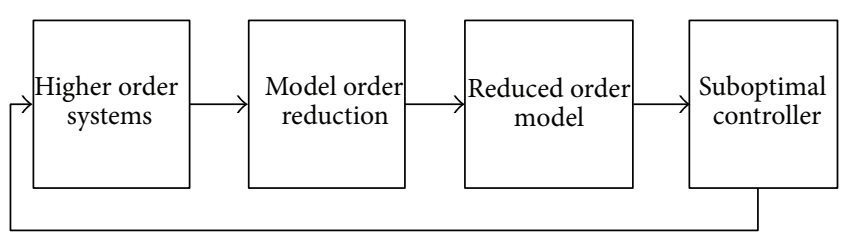

FIGURE 2: Flow diagram of suboptimal control design.

The control action of the plant is given as

$$
u(t)=-R^{-1} B^{T} P x=-k^{T} x,
$$

where $P$ is the symmetric positive definite matrix whose elements may be obtained by solving the following matrix Riccati equation:

$$
A^{T} P+P A-P B R^{-1} B^{T} P+Q=0 .
$$

The closed loop transfer function with controller is obtained as

$$
\begin{aligned}
T *(s) & =C^{T}\left[s I-A+B k^{T}\right]^{-1} B \\
& =\frac{s^{n-1}+a_{1} s^{n-2}+\cdots+a_{n-2} s^{2}+a_{n-1} s+a_{n}}{s^{n}+b_{1} s^{n-1}+\cdots+b_{n-2} s^{2}+b_{n-1} s+b_{n}} .
\end{aligned}
$$

2.1. Model Order Reduction Procedure by Padé Approximation. Consider a $n$th order system [5] as

$$
\begin{aligned}
G_{n}(s)= & \frac{a_{1} s^{n-1}+a_{2} s^{n-2}+\cdots+a_{n}}{s^{n}+b_{1} s^{n-1}+\cdots+b_{n}} \\
= & t_{0}+t_{1} s+t_{2} s^{2}+\cdots \\
& (\text { expansion around } s=0) \\
= & M_{1} s^{-1}+M_{2} s^{-2}+\cdots \\
& (\text { expansion around } s=\infty) .
\end{aligned}
$$

It is desired to obtain a stable $r$ th order approximant as

$$
\begin{aligned}
G_{r}(s)= & \frac{a_{1}^{\prime} s^{n-1}+a_{2}^{\prime} s^{n-2}+\cdots+a_{r}^{\prime}}{s^{n}+b_{1}^{\prime} s^{n-1}+\cdots+b_{r}^{\prime}} \\
= & t_{0}^{\prime}+t_{1}^{\prime} s+t_{2}^{\prime} s^{2}+\cdots \\
& (\text { expansion around } s=0) \\
= & M_{1}^{\prime} s^{-1}+M_{2}^{\prime} s^{-2}+\cdots \\
& (\text { expansion around } s=\infty) .
\end{aligned}
$$

It is easy to verify that the following equations hold true:

$$
\begin{gathered}
t_{1}^{\prime}=\frac{a_{r}^{\prime}}{b_{r}^{\prime}}, \quad i=1, \\
t_{i}^{\prime}=a_{r+1-i}^{\prime}-\sum_{j}^{i-1}\left(t_{i}^{\prime} b_{r+j-i}^{\prime}\right) b_{r}^{\prime-1}, \quad i=2,3, \ldots,
\end{gathered}
$$

and $a_{i}^{\prime}=0$ for $i \leq 0 ; b_{0}^{\prime}=1 ; b_{i}^{\prime}=0$ for $i \leq-1$;

$$
\begin{gathered}
M_{i}^{\prime}= \begin{cases}a_{i}^{\prime}, & i=1, \\
a_{i}^{\prime}-\sum_{j=1}^{i-1} M_{j}^{\prime} b_{i-j}^{\prime}, & i=2,3, \ldots,\end{cases} \\
a_{i}=b_{i}=0 \text { for } i=r+1, r+2 .
\end{gathered}
$$

We seek a reduced order model so as to satisfy

$$
\begin{gathered}
t_{0}^{\prime}=t_{0} \Longrightarrow a_{r+1-i}^{\prime}=\sum_{j=1}^{i} t_{j} b_{i-j}^{\prime}, \quad i \in\{1,2, \ldots, \lambda\} \\
M_{i}^{\prime}=M_{i} \Longrightarrow a_{i}^{\prime}=\sum_{j=1}^{i} M_{j} b_{i-j}^{\prime}, \quad i \in\{1,2, \ldots, \gamma\},
\end{gathered}
$$

where $\lambda+\gamma=2 r$.

Using (8)-(16) the reduced order model is obtained.

2.2. Suboptimal Controller Design Procedure. The suboptimal controller [4] is designed with the help of reduced order model by choosing the optimal gain matrix in such a way that

$$
K_{\text {sub }}=\left[\begin{array}{llll}
K_{\text {red }} & 0 & \cdots & 0
\end{array}\right],
$$

where $K_{\text {red }}$ is optimal gain matrix of reduced order model, obtained using (3) and (4). Finally this $K_{\text {sub }}$ is applied to higher order system in order to control the plant (higher order system).

\subsection{Flow Diagram of Suboptimal Controller Design. See Figure 2.}

2.4. Comparison of Responses of Optimal and Suboptimal Controller. Using (5) one can try to determine the closed loop transfer functions with optimal controller and suboptimal controller with the gain matrices $K_{\text {opt }}$ and $K_{\text {sub }}$, respectively being derived for comparison.

\section{Numerical Examples}

The step-by-step procedure to obtain reduced the order model is explained with the help of the example presented below and procedure is derived for suboptimal controller.

Example 1. Consider the following 4th order system [3]:

$$
G_{4}(s)=\frac{s^{3}+7 s^{2}+24 s+24}{s^{4}+10 s^{3}+35 s^{2}+50 s+24} .
$$




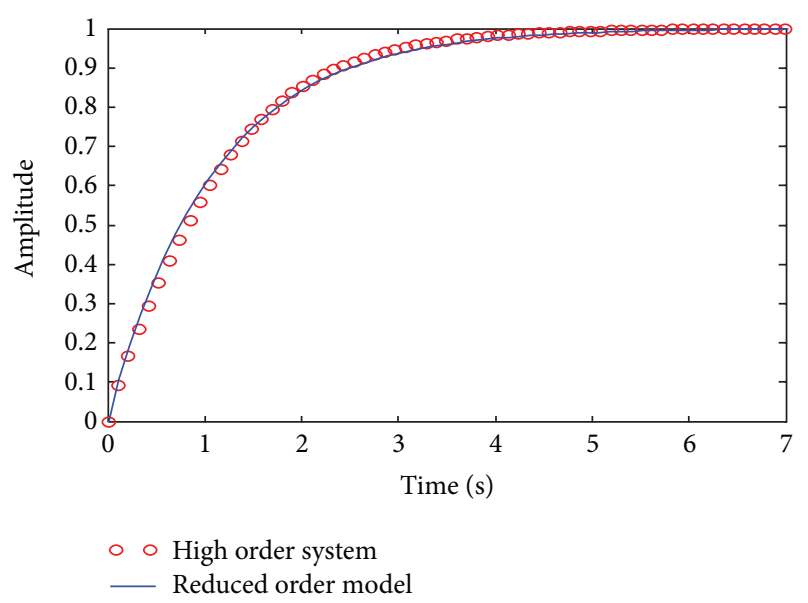

FIGURE 3: Step response of higher order system and reduced order model.

Suppose that reduced order model of the form

$$
G_{2}(s)=\frac{a_{1}^{\prime} s+a_{2}^{\prime}}{s^{2}+b_{1}^{\prime} s+b_{2}^{\prime}}
$$

is required.

Reduction Steps. The approximant can systematically be derived in the following steps for second order approximant.

Step 1. Expand (12) in power series around $s=0$ and $s=\infty$ :

$$
\begin{aligned}
G_{4}(s) & =\frac{s^{3}+7 s^{2}+24 s+24}{s^{4}+10 s^{3}+35 s^{2}+50 s+24} \\
& =1-1.0833 s+1.0902 s^{2}-1.0664 s^{3}+\cdots \\
& =1 s^{-1}-3 s^{-2}+19 s^{-3}-111 s^{-4}+571 s^{-5}+\cdots .
\end{aligned}
$$

Step 2. Expand (13) in power series around $s=0$ and $s=\infty$ :

$$
\begin{aligned}
G_{2}(s) & =\frac{a_{1}^{\prime} s+a_{2}^{\prime}}{s^{2}+b_{1}^{\prime} s+b_{2}^{\prime}} \\
& =t_{1}^{\prime}+t_{2}^{\prime} s+t_{3}^{\prime} s^{2}+\cdots \\
& =M_{1}^{\prime} s^{-1}+M_{2}^{\prime} s^{-2}+M_{3}^{\prime} s^{-3}+\cdots
\end{aligned}
$$

The time moment and Markov parameter expansion can obtain about $s=0$ and $s=\infty$.

Step 3. For perfect matching we have to match the $(2 r)$ term:

$$
\begin{gathered}
t_{1}=t_{1}^{\prime}, \\
M_{1}=M_{1}^{\prime}, \\
t_{2}=t_{2}^{\prime}, \\
M_{2}=M_{2}^{\prime} .
\end{gathered}
$$

Step 4. By considering the above assumptions we can get the second order reduced model by solving the following and get the variables of reduced order model (13),

$$
\begin{gathered}
t_{1}^{\prime}=\frac{a_{2}^{\prime}}{b_{2}^{\prime}}, \\
t_{2}^{\prime}=\frac{a_{1}^{\prime}-t_{1}^{\prime} b_{1}^{\prime}}{b_{2}^{\prime}}, \\
M_{1}^{\prime}=a_{1}^{\prime}, \\
M_{2}^{\prime}=a_{2}^{\prime}-M_{1}^{\prime} b_{1}^{\prime} .
\end{gathered}
$$

But for better overall time response approximation, equal emphasis is given to both time moments and Markov parameters. For second order reduced model, we have to match $2 r$ terms and satisfy $\lambda+\gamma=2 r$; here $r=2$ and consider $\lambda=\gamma=2$.

By solving (17) we get

$$
\begin{gathered}
a_{1}^{\prime}=1, \\
a_{2}^{\prime}=24.0096, \\
b_{1}^{\prime}=27.0096, \\
b_{2}^{\prime}=24.0096
\end{gathered}
$$

and require the reduced order model obtained as

$$
G_{2}(s)=\frac{s+24.0096}{s^{2}+27.0096 s+24.0096} .
$$

The step response of higher order system (12) and reduced order model (19) is plotted as shown in Figure 3.

Model (19) is seen to be a closed approximant to (12).

The optimal controller and suboptimal controller can be designed for a particular performance index by solving Riccati equation (4).

The optimal controller for actual higher order system (12) is obtained by using (3)-(4) as

$$
K_{\mathrm{opt}}=\left[\begin{array}{llll}
0.0626 & 0.1275 & 0.0857 & 0.0208
\end{array}\right] \text {, }
$$

and the transfer function with optimal controller is obtained by putting the value of gain matrix (20) in (5) as

$$
T_{K_{\mathrm{opt}}}(s)=\frac{s^{3}+7 s^{2}+24 s+24}{s^{4}+10.06 s^{3}+35.13 s^{2}+50.86 s+24.02} .
$$

Similarly, the optimal gain matrix for reduced order model (19) by using (3) and (4) is as follows:

$$
K_{\mathrm{red}}=\left[\begin{array}{ll}
0.0193 & 0.0208
\end{array}\right] \text {. }
$$

The closed loop transfer function of reduced order model (19) is obtained by putting (22) in (5) as

$$
T_{K_{\text {red }}}(s)=\frac{s+24.01}{s^{2}+27.03 s+24.03} .
$$




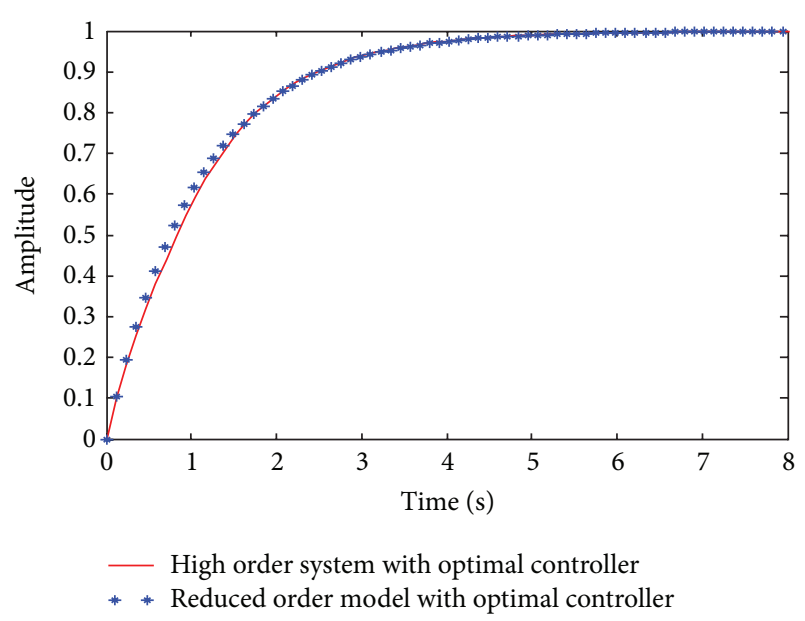

FIGURE 4: Step response of higher order and reduced order model with optimal controller.

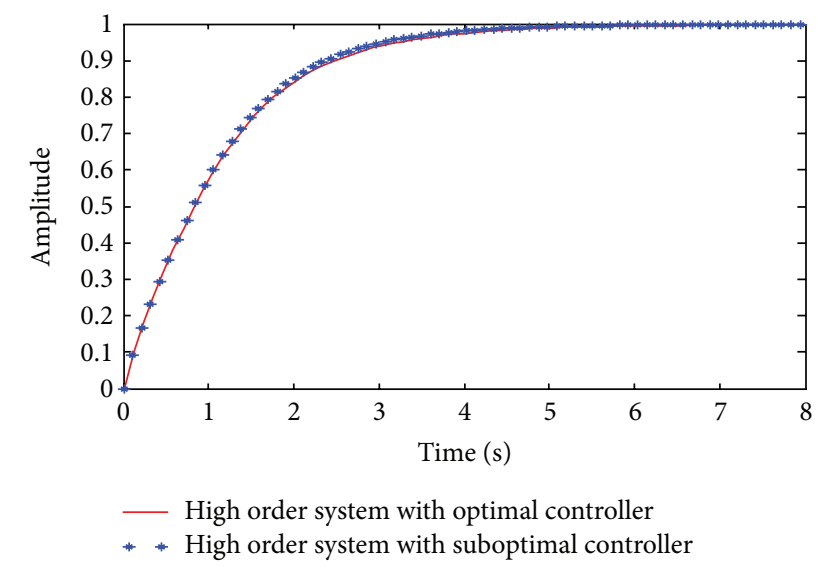

FIGURE 5: The step response of higher order system with optimal and with suboptimal controller.

The step response of higher order system with optimal controller (21) and reduced order model with optimal controller (23) is shown in Figure 4.

It is found that (23) is a closed approximation to (21).

The suboptimal controller (11) is obtained with the help of reduced order model using $K_{\text {sub }}=\left[\begin{array}{llll}K_{\text {red }} & 0 & \cdots & 0\end{array}\right]$.

Thus $K_{\text {sub }}$ takes the following form:

$$
K_{\text {sub }}=\left[\begin{array}{llll}
0.0193 & 0.0208 & 0 & 0
\end{array}\right] .
$$

The transfer function with suboptimal controller is obtained by putting the value of (24) in (5) as

$$
\widetilde{T}_{\text {Ksub }}(s)=\frac{s^{3}+7 s^{2}+24 s+24}{s^{4}+10.02 s^{3}+35.02 s^{2}+50 s+24} .
$$

The step response of higher order system with optimal control (21) and with suboptimal controller (25) is shown in Figure 5.

The step responses of higher order system with optimal controller (21) and with suboptimal controller (25) are shown

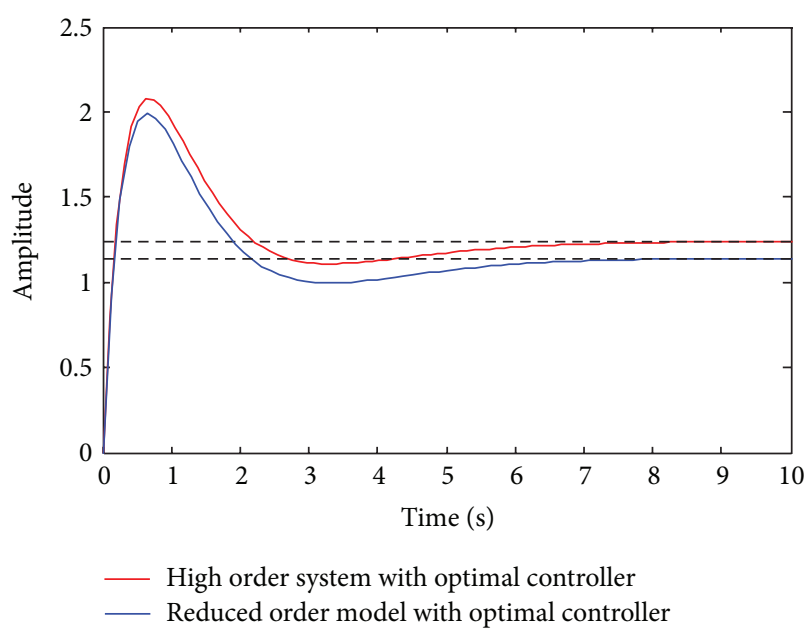

FIGURE 6: Step response of higher order system and reduced order model with optimal controller.

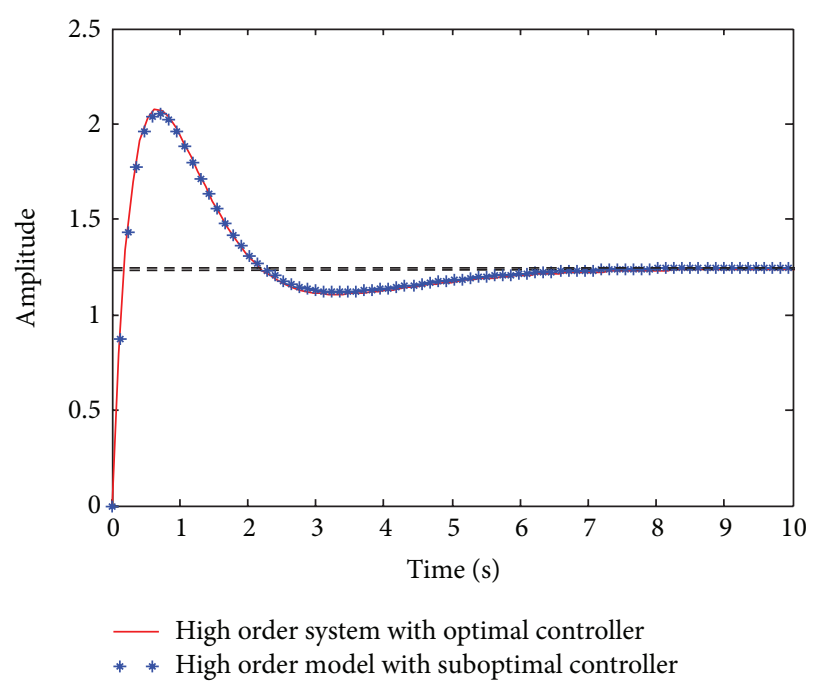

FIGURE 7: The step response of higher order system with optimal and suboptimal controller.

in Figure 5. The response of suboptimal controller (25) is seen identical to (21). This shows that we can control the plant with the help of only measurable states which reduces the complexity and cost.

Example 2. Consider the higher order system [6]

$$
G_{4}(s)=\frac{9 s^{3}+42 s^{2}+31 s+10}{s^{4}+8 s^{3}+21 s^{2}+22 s+8}
$$

The optimal controller for (26) is obtained using (3) and (4) as

$$
K_{\mathrm{opt}}=\left[\begin{array}{llll}
0.0821 & 0.1603 & 0.1405 & 0.0623
\end{array}\right] .
$$

The closed loop transfer function using (5) is expressed as

$$
T_{\text {opt }}(s)=\frac{9 s^{3}+42 s^{2}+31 s+10}{s^{4}+8.082 s^{3}+21.16 s^{2}+22.14 s+8.062} .
$$


It is assumed that one state is not available for measurement. Thus a reduced order model $(r=3)$ is desired which takes the following form:

$$
G_{3}(s)=\frac{9 s^{2}+7.145 s+2.735}{s^{3}+4.127 s^{2}+4.95 s+2.188} .
$$

And the optimal gain matrix for (29) is obtained with the help of (3) and (4) as

$$
K_{\text {red }}=\left[\begin{array}{lll}
0.2053 & 0.3686 & 0.2177
\end{array}\right] .
$$

The closed loop transfer function of (29) with optimal controller is obtained by putting (30) into (5) as

$$
T_{\text {red }}(s)=\frac{9 s^{2}+7.145 s+2.735}{s^{3}+4.333 s^{2}+5.319 s+2.406} .
$$

The comparison of the result of step responses of higher order system with optimal controller (28) and reduced order model with optimal controller (31) is shown in Figure 6.

It is clear that the step response of higher order system with optimal controller (28) and reduced order model with optimal controller (31) is very close to identical.

The suboptimal gain matrix is obtained for the system (26) with the help of (11) and (30) and is expressed as

$$
K_{\text {sub }}=\left[\begin{array}{llll}
0.2053 & 0.3686 & 0.2177 & 0
\end{array}\right] .
$$

The closed loop transfer function of higher order system (26) with suboptimal controller is obtained using (32) and (5) as

$$
\widetilde{T}_{K_{\text {sub }}}(s)=\frac{9 s^{3}+42 s^{2}+31 s+10}{s^{4}+8.205 s^{3}+21.37 s^{2}+22.22 s+8} .
$$

The step responses of (28) and (33) are shown in Figure 7 and are found identical in transient as well as steady state region.

The results of the two examples signify that the suboptimal control provides a good platform for controlling the higher order system and reduces the complexity and cost of control action.

For purpose of comparison, the suboptimal controller is also derived using the technique in [4]. The result obtained in [4] shows remarkable deviation in the transient part which confirms the observation of [4] that, for better overall time response approximation, matching of both time moments and Markov parameters is required.

\section{Conclusion}

A design technique of suboptimal controller using partial feedback is proposed. The method is based on the Padé approximation technique (matching of both time moments and Markov parameters) for model order reduction which results in better overall time response approximation. In this method, the suboptimal controller is first derived for reduced order model and then implemented for higher order plant. This reduces the design complexity and cost of control action.
The method also avoids use of observer or Kalman filter for reconstruction of missing states.

In this paper equal emphasis is given for matching of time moments and Markov parameters; however, the optimal choice of Markov parameters and time moments to be matched remains unresolved and it is open to further investigation.

\section{Conflict of Interests}

The authors declare that there is no conflict of interests regarding the publication of the paper.

\section{References}

[1] G. Bengtsson and S. Lindahl, "A design scheme for incomplete state or output feedback with applications to boiler and power system control," Automatica, vol. 10, no. 1, pp. 15-30, 1974.

[2] T. C. Hsia, "An approach for incomplete state feedback control systems design," IEEE Transactions on Automatic Control, vol. AC-17, pp. 383-386, 1972.

[3] V. P. Singh, P. Chaubey, and D. Chandra, "Model order reduction of continuous time systems using pole clustering and Chebyshev polynomials," in Proceedings of the IEEE Students Conference on Engineering and Systems (SCES '12), 2012.

[4] J. Pal, "Suboptimal control using Padé approximation technique," IEEE Transactions on Automatic Control, vol. AC-25, no. 5, 1980.

[5] V. Singh, D. Chandra, and H. Kar, "Improved Routh-Padé approximants: a computer-aided approach," IEEE Transactions on Automatic Control, vol. 49, no. 2, pp. 292-296, 2004.

[6] D. Chandra, An investigation into Routh's Padé approximants [Ph.D. thesis], Department of Electrical Engineering, Motilal Nehru National Institute of Technology, Allahabad, India.

[7] D. S. Janardhanan, "Model Order Reduction and Controller Design," http://web.iitd.ac.in/ janas/courses/material/eel879/ sp_topics_01.pdf. 

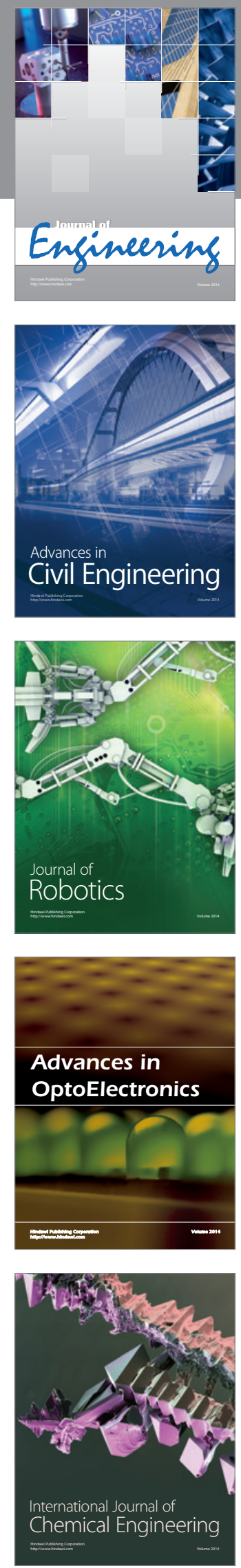

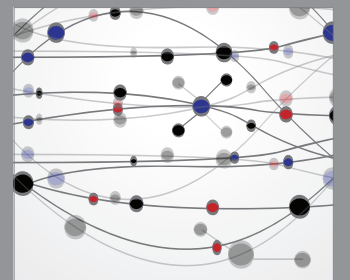

The Scientific World Journal
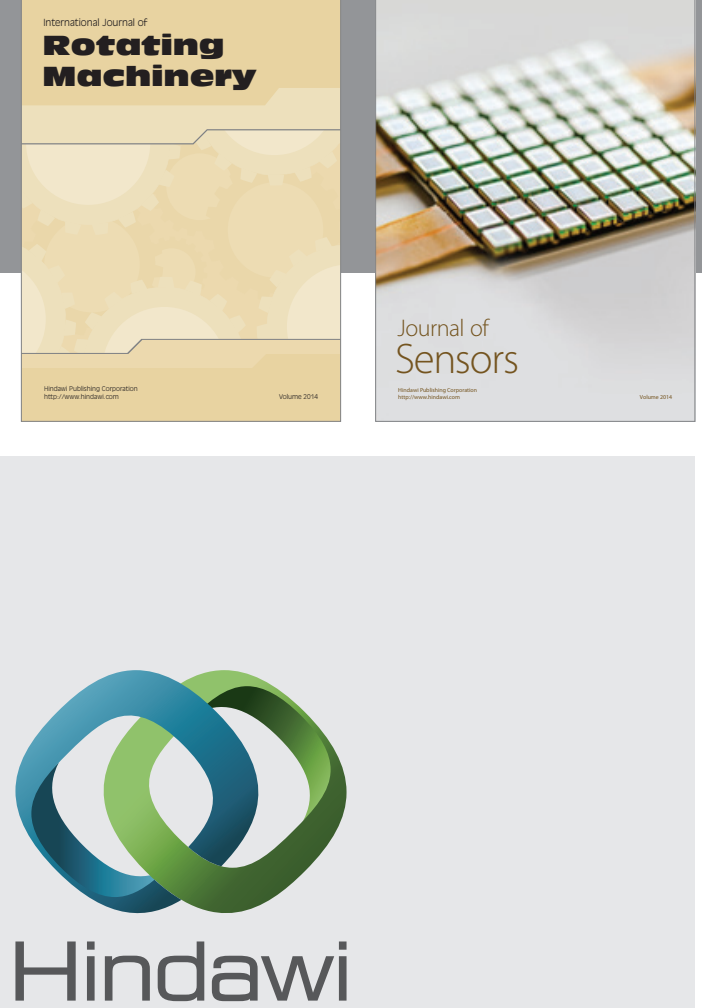

Submit your manuscripts at http://www.hindawi.com
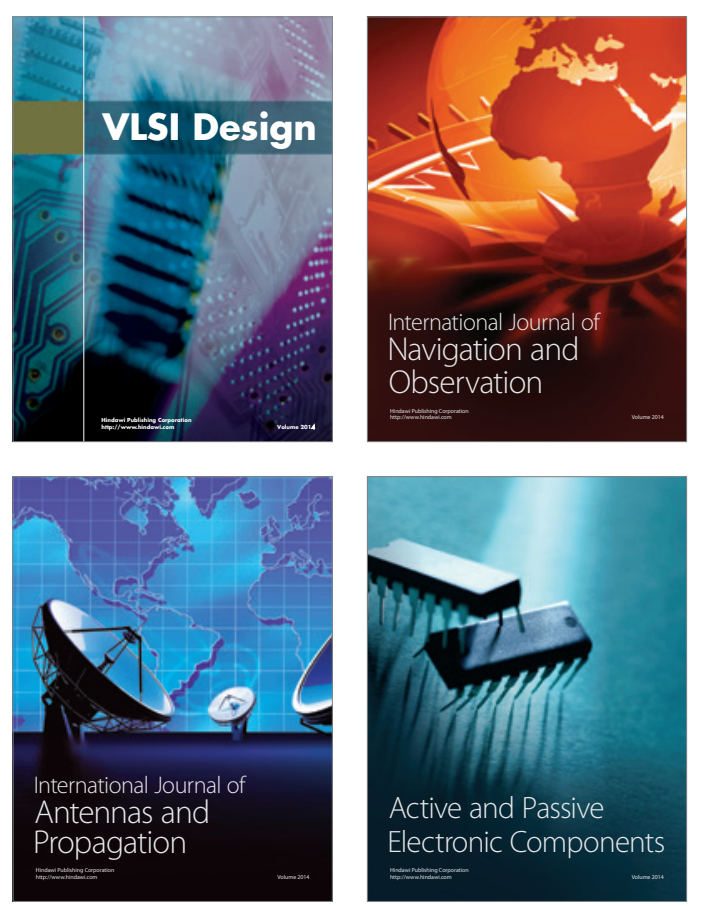
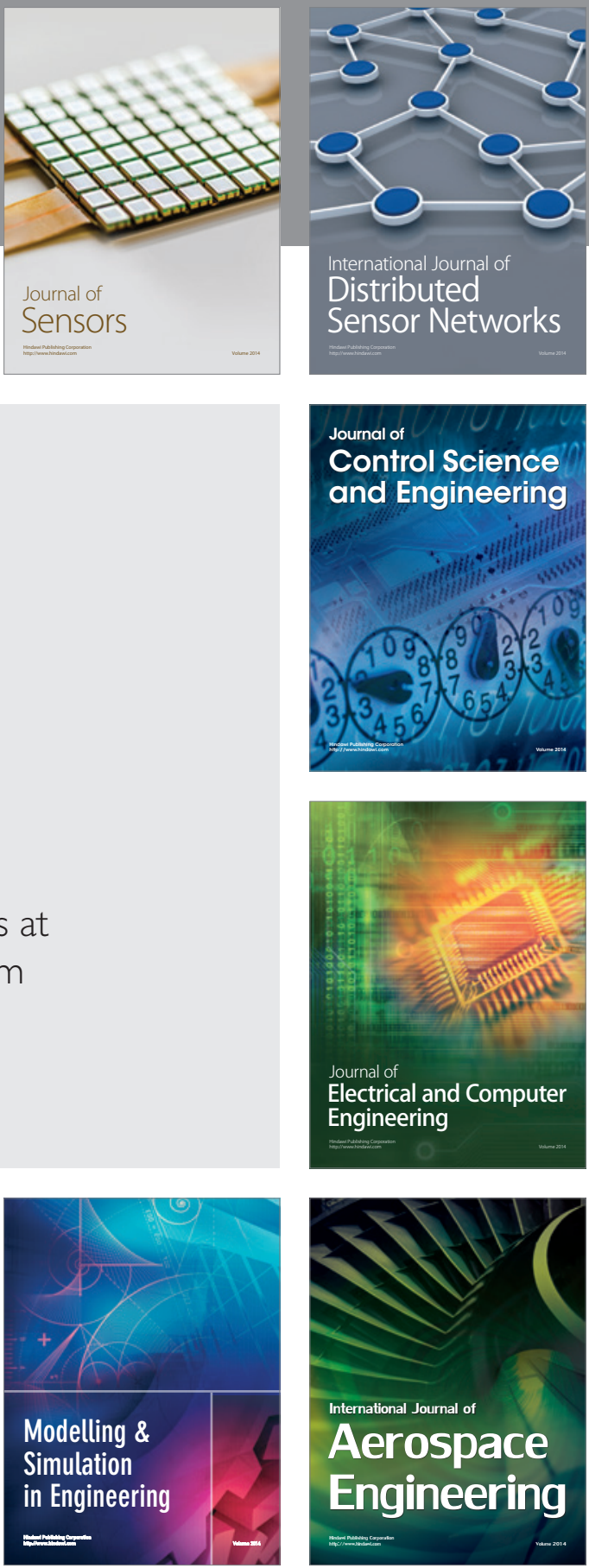

Journal of

Control Science

and Engineering
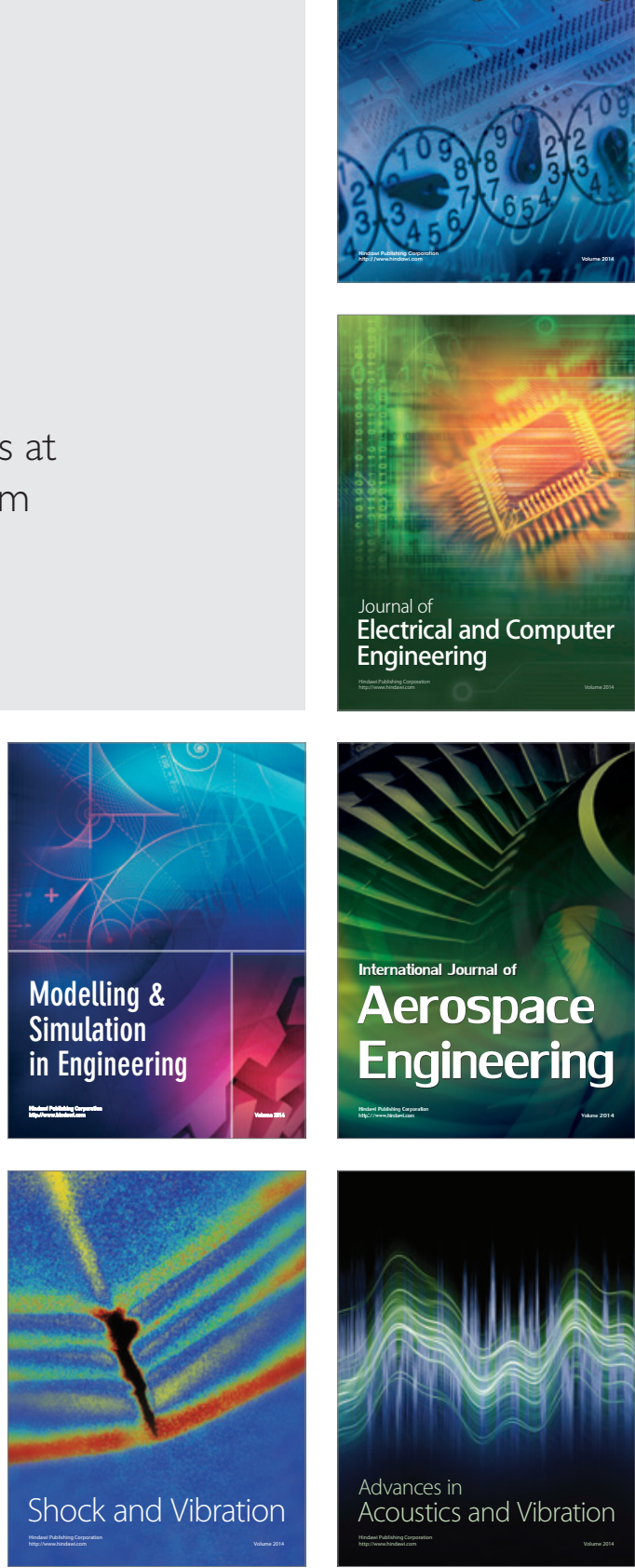